\title{
Optimización de costos de producción agregada en empresas del sector textil
}

\section{Optimization of aggregate production costs in textile companies}

\author{
Emiro Antonio Campo ${ }^{1,2}$ Jose Alejandro Cano ${ }^{2 *} \quad$ Rodrigo Andrés Gómez-Montoya ${ }^{1,3}$ \\ Recibido 19 de julio de 2018, aceptado 25 de marzo de 2019 \\ Received: July 19, 2018 Accepted: March 25, 2019
}

\begin{abstract}
RESUMEN
Esta investigación tiene como objetivo desarrollar un modelo de planificación de producción agregada para generar estrategias de producción óptimas en el mediano plazo para empresas del sector textil. Para esto se desarrolla un modelo de planificación de producción agregada denominado PLAG, que minimiza los costos de mano de obra, costos de gestión de inventarios, y costos de subcontratación de producción. A diferencia de otros modelos de la literatura, el modelo PLAG tiene en cuenta características del sector textil relacionadas con la contracción de tela, pérdidas por manipulación del producto en el proceso, eficiencia de empleados nuevos, tiempos de entrenamiento y capacitación, y subcontratación de procesos de manufactura, lo cual hace que sea un modelo completo y eficaz para las empresas del sector textil. El modelo propuesto se programa y ejecuta en GAMS, apoyándose de una interface en MSExcel, con lo cual se generan estrategias de capacidad de producción para el mejoramiento del proceso productivo y la optimización del plan de producción.
\end{abstract}

Palabras clave: Optimización, costos de producción, planificación agregada, textiles.

\begin{abstract}
This article aims to optimize the aggregate production costs in textile companies sector through production strategies in the medium term. For this, an aggregate production planning model called PLAG is developed, which minimizes labor costs, inventory management costs, and production subcontracting costs. Unlike other models in the literature, the PLAG model considers characteristics of the textile sector related to fabric shrinkage, wastes due to product handling, new employees' efficiency, training time, and manufacturing processes subcontracting, which makes it a comprehensive and effective model for companies in the textile industry. The proposed model is programmed and executed in GAMS, supported by an interface in MSExcel, which generates strategies for the improvement of the production capacity and the optimization of the production plan.
\end{abstract}

Keywords: Optimization, production costs, aggregate planning, textiles.

1 ESACS - Escuela Superior en Administración de Cadena de Suministro. Medellín, Colombia. E-mail: ecampo@udem.edu.co; academia@esacsvirtual.com

2 Universidad de Medellín. Facultad de Ciencias Económicas y Administrativas. Medellín, Colombia. E-mail: jacano@udem.edu.co

3 Politécnico Colombiano Jaime Isaza Cadavid. Facultad de Ingeniería. Medellín, Colombia. E-mail: ragomez@elpoli.edu.co

* Autor de correspondencia: jacano@udem.edu.co 


\section{INTRODUCCIÓN}

Las empresas constantemente se enfrentan al reto de desarrollar buenas estrategias productivas para generar ventajas competitivas y garantizar su sostenibilidad en el tiempo [1], y para lograrlo enfrentan el problema de optimizar, ecualizar y adaptar la capacidad de la empresa a las demandas de los clientes [2]. La planificación de producción agregada se considera como una herramienta de planificación de capacidad de mediano y largo plazo que sugiere estrategias de producción con el fin de alcanzar la demanda pronosticada, teniendo en cuenta las restricciones de capacidad existentes [3], brindando una guía al fabricante respecto a estrategias eficientes de producción y abastecimiento [4]. En este sentido, la planificación de producción agregada determina la capacidad de fabricación de productos para satisfacer la demanda en el mediano plazo [5], evaluando configuraciones de diseño y escenarios de fabricación que maximizan beneficios y/o minimizan costos a través de niveles óptimos de producción, capacidad, subcontratación, inventario y faltantes [6].

Para dar solución a los problemas de planificación de la producción agregada, existen estrategias de equilibrio, alcance y mixtas. Las estrategias de equilibrio o nivel mantienen estables la cantidad de mano de obra y tasa de producción, y para cubrir variaciones de demanda se acude al uso de inventarios, a portafolios de productos con demandas complementarias, a influenciar en el incremento de la demanda, además de la generación de órdenes pendientes [7]. Las estrategias de alcance igualan la tasa de producción con la tasa de demanda en cada periodo de tiempo, a través de contratación y despido de mano de obra, uso de horas extra, subcontratación de producción, uso de trabajadores medio tiempo, entre otras. Las estrategias mixtas combinan las opciones planteadas por las estrategias de equilibrio y alcance, con el fin de generar un plan de producción óptimo, que satisface la demanda y otras políticas empresariales al menor costo $[1,8]$.

La complejidad generada por la formulación de estrategias mixtas, hace que sea necesario el uso de modelos de optimización basados en programación matemática, que pueden ser modelos de programación lineal con modelos de transporte [9], programación lineal entera mixta (MILP) [10,11], programación lineal entera mixta multicriterio (MCMILP) [12], y programación no lineal (NLP) [13], entre otros.

En la literatura relacionada con modelación de problemas de producción agregada, algunos estudios plantean funciones objetivo relacionados con costos totales, fluctuaciones de mano de obra, órdenes tardías, beneficios y utilidades, uso de equipos, nivel de servicio, calidad, entre otros $[6,11,12,14$, 15], en los cuales se plantean funciones objetivo relacionados con costos totales, fluctuaciones de mano de obra, ordenes tardías, beneficios y utilidades, uso de equipos, nivel de servicio, calidad, entre otros. De igual forma, encuentran parámetros, variables y restricciones relacionadas con el tamaño de mano de obra, contrataciones y despidos, tasas de aprendizaje de mano de obra, horas laborales y restricciones legales, nivel y capacidad de producción, subcontratación de producción, múltiples plantas de producción, capacidad de almacenamiento y niveles de inventario, mantenimiento de máquinas, tiempos de alistamientos y montajes, demanda de clientes y manejo de efectivo.

Respecto a métodos de optimización en sistemas de producción textil, se encuentra que Plonka y Oginski [16] aplican una metodología basada en expertos y múltiples criterios para mejorar la calidad y minimizar los costos de fabricación de husos, sin embargo, no se enfocan en planificar el proceso productivo. Zhang, Chang, Song y Wu [2] proponen un modelo multiobjetivo para el problema de programación de producción de corto plazo, con el fin de minimizar los costos de tardanza y el nivel de emisión de polución en el proceso de teñido de tela. Cabe resaltar que esta programación es a corto plazo y solo se programa el proceso cuello de botella, en este caso el proceso de teñido, más no todos los procesos textiles. Por su parte, Mercado, Fontalvo y Granadillo [17] presentan un análisis comparativo de las cadenas productivas del sector textil-confecciones de la provincia de Jiangsu-China y el departamento del Atlántico-Colombia para establecer estrategias y políticas que mejoren la productividad del sector textil, así como también identificar diferencias de producción existentes entre estas cadenas productivas. Lopes, Varela, Trojanowska y Machado [18] presentan un caso de estudio del flujo de producción en una empresa textil de Portugal que se basa en la reorganización de la distribución de planta y puestos de trabajo, 
sin embargo, no optimizan como tal el proceso productivo. Campo, Cano y Gómez-Montoya [19] proponen un modelo programación lineal para planificar la producción anual en empresas del sector textil para minimizar costos de mano de obra e inventarios, considerando contracción de tela, desperdicios, eficiencia de nuevos empleados, capacitación y entrenamiento.

Por lo tanto, existen muy pocos artículos en la literatura que aborden modelos de planificación agregada para empresas de la industria textil, la cual requiere estrategias para el mejoramiento de la productividad y competitividad [17, 18, 20], que además de tener en cuenta variables de desperdicios y contracción de tela por línea y proceso, días requeridos para el entrenamiento de empleados, y la eficiencia de nuevos empleados en cada proceso, tengan en cuenta la subcontratación de procesos de manufactura, lo cual es una práctica común dentro de este sector industrial. Por lo tanto, este artículo tiene como objetivo desarrollar un modelo de planificación de producción agregada para generar estrategias de producción óptimas en el mediano plazo para empresas del sector textil, teniendo en cuenta subcontratación de uno o varios procesos de manufactura. Es por esto que en la sección 2 se presenta una formulación algebraica para modelar la planificación agregada considerando condiciones específicas de las empresas de la industria textil. En la sección 3 se valida el modelo a través de su aplicación en una empresa del sector textil. La sección 4 presentan los resultados arrojados por el modelo y se analiza la solución óptima obtenida a través de gráficos, precios duales y análisis de sensibilidad. Finalmente, en la sección 5 se muestran las principales conclusiones y aportes de esta investigación.

\section{FORMULACIÓN MATEMÁTICA}

El tipo de empresas en el cual se enfoca la investigación se dedican a la fabricación de productos que presentan altos índices de rotación. Este tipo de empresas cuentan con varias líneas de productos, las cuales son de consumo permanente por parte de sus clientes. La fabricación de cada línea de producto involucra a su vez diferentes procesos de transformación.

El modelo a desarrollar tiene como objetivo determinar un plan de producción agregado anual para minimizar los costos totales (costos de mano de obra, gestión de inventarios, y subcontratación de procesos) y asegurar el cumplimiento de restricciones específicas (demanda de cada línea de producto, capacidad de producción, almacenamiento por proceso, y eficiencia operativa). Adicional a esto, el modelo propuesto debe establecer con anticipación la necesidad de subcontratar capacidad en procesos para garantizar capacidad de producción en las plantas externas durante el horizonte de planificación. Igualmente, el modelo debe tener en cuenta la eficiencia de nuevos empleados, debido a que dedican una parte inicial de su contrato laboral a recibir capacitaciones y entrenamientos, $\mathrm{e}$ igualmente presentan menor rendimiento debido a la poca experiencia en el cargo asignado. También debe incluirse en el plan agregado de producción los desperdicios que se generan entre procesos, que para el caso del sector textil son causados por la contracción de la tela dentro de los procesos de la empresa y en las empresas subcontratadas.

Por políticas establecidas en muchas empresas, no se considera el uso de horas extras, y se suelen subcontratar algunos procesos, especialmente aquellos que no afectan significativamente la calidad del producto o pongan en riesgo secretos industriales. De igual forma, al tratarse de un plan de producción de mediano plazo, no se consideran directamente detalles operativos relacionados con tiempos de alistamiento, montajes y mantenimiento de máquinas; sin embargo, con base en estudios de métodos y tiempos estos factores se incluyen de forma proporcional a los tiempos de operación de cada línea de producto en cada proceso de manufactura.

Por otro lado, cuando un empleado nuevo se integra a un proceso productivo, este requiere recibir capacitación y entrenamiento, lo cual requiere una cantidad de tiempo en días según las características y estándares específicos de cada proceso. Durante el tiempo de entrenamiento los empleados no generan producción, solo acompañan los procesos, por lo cual es importante tener en cuenta estos factores para calcular el nivel de producción real cuando se contratan empleados a un proceso operativo.

Debido a la complejidad generada por los objetivos, condiciones particulares a cumplir para obtener un plan factible, y a la necesidad de generar un plan de producción óptimo, se acudirá al uso de la programación matemática para crear así un modelo de 
programación lineal para la planificación agregada, que se denominará como el modelo PLAG.

Es de aclarar, que en la revisión de literatura se identificaron modelos con algunos de los elementos que se requieren para modelar el proceso productivo de la empresa en estudio $[6,11,12,14,15]$, pero estos modelos no se adaptan completamente a las necesidades y realidades empresariales anteriormente mencionadas. En la Tabla 1 se presentan los índices, parámetros, coeficientes y variables del modelo PLAG, los cuales permiten su planteamiento conceptual o modelación, considerando la posibilidad de subcontratar diversos procesos de manufactura.

Una vez definidos los elementos que permiten generar un modelo matemático del problema real de planificación agregada, se procede a elaborar la función objetivo y restricciones de programación matemática, que componen al modelo PLAG.

$$
\begin{aligned}
& \operatorname{Min} Z= \sum_{t=1}^{T} \sum_{i=1}^{I}\left[C E_{i} \times\left(X_{i, t}+N X_{i, t} \times \frac{D E_{i}}{30}\right)+\right. \\
& C A C \times X N_{i, t}+C A D \times+X N_{i, t} \mid+ \\
& \sum_{t=1}^{T} \sum_{i=1}^{I} \sum_{l=1}^{L}\left[\left(C A_{i, t} \times S_{l, i, t}\right)+\left(C S C_{l, i, t} \times Y_{i, i, t}\right)\right]
\end{aligned}
$$

La función objetivo del modelo PLAG, representada en la ecuación (1), busca minimizar los costos totales del plan de producción, representados por los costos de mano de obra (costo mensual de empleados en cada proceso, costos de contratación de empleados al inicio del mes, y costos de despido de empleados al final del mes), costos de gestión de inventarios, y costos de subcontratación de producción. Los costos de mano de obra se representan por el costo mensual de un empleado por proceso multiplicado por el número de empleados utilizados en dicho proceso en un mes, más el tiempo de entrenamiento

\begin{tabular}{|c|c|c|}
\hline \multicolumn{2}{|c|}{$\begin{array}{c}\text { Índices, Parámetros } \\
\text { y Variables }\end{array}$} & Definición \\
\hline \multirow{3}{*}{ Índices } & I & Número de procesos $(i=1, \ldots, \mathrm{I})$ \\
\hline & $\mathrm{T}$ & Número de meses $(t=1, \ldots, \mathrm{T})$ \\
\hline & $\mathrm{L}$ & Número de líneas $(l=1, \ldots, \mathrm{L})$ \\
\hline \multirow{16}{*}{ Parámetros } & $\mathrm{CE}_{i}$ & Costo mensual por empleado en el proceso $i$ (\$/mes) \\
\hline & $\mathrm{CSC}_{i, l, t}$ & Costo de subcontratar el proceso $i$ en línea $l$ en el mes $t(\$ /$ metro $)$ \\
\hline & $\mathrm{YD}_{i}$ & Parámetro binario que representa la posibilidad de subcontratar el proceso $i$ \\
\hline & $\mathrm{CA}_{i, t}$ & Costo de gestión de inventario en el proceso $i$ en el mes $t(\$ /$ metro $)$ \\
\hline & $\mathrm{A}_{i}$ & Capacidad de almacenamiento en proceso $i$ (miles de metros / mes) \\
\hline & $\mathrm{PM}_{i}$ & Capacidad máxima de producción del proceso $i$ (miles de metros / mes) \\
\hline & $\mathrm{DT}_{l, i}$ & Desperdicio de tela de la línea $l$ en el proceso $i$ (adimensional) \\
\hline & DTY $_{l, i}$ & Desperdicio de tela de la línea $l$ en el proceso subcontratado $i$ (adimensional) \\
\hline & $\mathrm{D}_{l, t}$ & Demanda esperada de tela de la línea $l$ en el mes $t$ (metros) \\
\hline & $\mathrm{DE}_{i}$ & Días de entrenamiento necesarios para empleado nuevo en el proceso $i$ (días) \\
\hline & $\mathrm{E}_{i}$ & Porcentaje de eficiencia de empleado nuevo en el proceso $i$ (adimensional) \\
\hline & $\mathrm{MH}_{l, i}$ & Producción de metros por hora de la línea $l$ en el proceso $i$ (metros/hora) \\
\hline & $\mathrm{HD}_{t}$ & Horas efectivas disponibles por empleado en el mes $t$ (horas) \\
\hline & $\mathrm{CAC}$ & Costo administrativo de contratación por empleado (\$) \\
\hline & CAD & Costo administrativo de despido por empleado (\$) \\
\hline & M & Un número muy grande (adimensional) \\
\hline \multirow{7}{*}{ Variables } & $\mathrm{H}_{l, i, t}$ & Horas de producción dedicadas a la línea $l$ en el proceso $i$ en el mes $t$ (horas) \\
\hline & $\mathrm{S}_{l, i, t}$ & Inventario final de la línea $l$ en el proceso $i$ en el mes $t$ (miles de metros) \\
\hline & $\mathrm{X}_{i, t}$ & Número de empleados en el proceso $i$ en el mes $t$ (empleados) \\
\hline & $\mathrm{XD}_{i, t}$ & Número de empleados despedidos en el proceso $i$ en el mes $t$ (empleados) \\
\hline & $\mathrm{XN}_{i, t}$ & Número de empleados contratados en el proceso $i$ en el mes $t$ (empleados) \\
\hline & $\mathrm{P}_{l, i, t}$ & Producción de tela de la línea $l$ en el proceso $i$ en el mes $t$ (metros) \\
\hline & $\mathrm{Y}_{l, i, t, t}$ & Producción de tela subcontratada de la línea $l$ en el proceso $i$ en el mes $t$ (metros) \\
\hline
\end{tabular}
de los mismos; y adicional a esto, se tiene en

Tabla 1. Definición de índices, parámetros y variables del modelo PLAG. 
cuenta el costo administrativo de contratar y despedir empleados. Los costos de gestión de inventario se calculan proporcionalmente al nivel de inventario mensual que se tenga en cada uno de los procesos. Los costos de subcontratación incluyen el procesamiento de productos en empresas externas, mas no el costo de las materias primas, que son suministradas por la empresa contratante.

$$
\begin{aligned}
& S_{l, I, t}=S_{l, I, t-1}+P_{l, I, t}+Y_{l, I, t}-D_{l, t} \text { para } \\
& l=1, \ldots, L t=1, \ldots, T \\
& S_{l, i, t}=S_{l, i, t-1}+P_{l, i, t}-\frac{P_{l, i+1, t}}{1-D T_{i, i}}+ \\
& Y_{l, i, t}-\frac{Y_{l, i+1, t}}{1-D T Y_{l, i}} \text { para } l=1, \ldots, \\
& L i=1, \ldots, I-1 \quad t=1, \ldots, T \\
& \sum_{i=1}^{L} S_{l, i, t} \leq A_{i} \text { para } i=1, \ldots, I \quad t=1, \ldots, T \\
& X_{i, t}=X_{i, t-1}+X N_{i, t}-X D_{i, t} \text { para } \\
& i=1, \ldots, I \quad t=1, \ldots, T \\
& P_{l, i, t}=H_{l, i, t} \times M H_{l, i} \text { para } \\
& l=1, \ldots, L \quad i=1, \ldots, I-1 \quad t=1, \ldots, T \\
& \sum_{i=1}^{L} H_{l, i, t}=H D_{t} \times\left(X_{i, t-1}+X N_{i, t} \times E_{i}-X D_{i, t}\right) \\
& \text { para } i=1, \ldots, I \quad t=1, \ldots, T \\
& \sum_{l=1}^{L} P_{i, i, t} \leq P M_{i} \text { para } i=1, \ldots, I t=1, \ldots, T \\
& Y_{l, i, t} \leq Y D_{i} \times M \text { para } l=1, \ldots, L i=1, \ldots, T \\
& H_{l, i, t} \geq 0, S_{l, i, t} \geq 0, X_{l, i, t} \geq 0, X D_{l, i, t} \geq \\
& 0, X N_{l, i, t} \geq 0, P_{l, i, t} \geq 0, Y_{l, i, t} \geq 0 \text { para } \\
& l=1, \ldots, L i=1, \ldots, I-1 t=1, \ldots, T
\end{aligned}
$$

La ecuación (2) garantiza el cumplimiento de la demanda de producto terminado para cada línea de producto; las restricciones de la ecuación (3) representan el cálculo de inventario final por periodo, proceso y línea; mientras que las restricciones (4) garantizan que no supere la capacidad máxima de almacenamiento en cada proceso. La ecuación (5) representa las ecuaciones de balance de mano de obra por contratación y despido. La producción en un periodo, proceso y línea de producto se representa en la ecuación (6); y en la ecuación (7) se calcula el número de horas mensuales disponibles en la planta de producción. Las restricciones de capacidad máxima de producción de planta se encuentran en la ecuación (8); mientras que la ecuación (9) garantiza que solo se puede subcontratar procesos que la empresa por políticas internas haya habilitado. Finalmente, la ecuación (10) representa las restricciones de no negatividad para las variables de salida y de decisión del modelo relacionadas con horas de producción, inventario final, contrataciones, despidos y nivel de producción.

Una vez modelado el problema de planificación de la producción agregada, se identifica que este corresponde a un modelo de programación lineal que no requiere manejar variables enteras, debido a la naturaleza de procesos productivos que fabrican de forma continua y no en unidades discretas, y para variables relacionadas con la mano de obra para el caso en que no se necesiten variables enteras de operarios, y según los resultados se decidirá acudir a opciones como contratar personal medio tiempo, redistribuir operarios con capacidad ociosa, aprovechar habilidades de polivalencia de empleados, asignar a otros procesos empleados con tiempo libre, entre otras.

\section{CASO DE ESTUDIO EN UNA EMPRESA TEXTIL}

Para validar el modelo PLAG, este se implementa en una empresa que maneja 3 líneas de producción, en cada línea de producción se llevan a cabo 5 procesos de manufactura (urdido, revisión de urdido, engomado, tejido y revisión de tejido), y se considera en la empresa que solo se puede subcontratar el proceso de tejido. De acuerdo con los pronósticos de demanda, las negociaciones realizadas con los clientes y proveedores de servicios de manufactura de la empresa, la Tabla 2 presenta para cada línea de producto la demanda mensual en un horizonte de planificación equivalente a un año. Igualmente, la Tabla 2 presenta las horas de trabajo disponibles por empleado al mes y los costos mensuales de 
subcontratación del proceso de tejeduría para cada línea de producto.

La Tabla 3 presenta los datos de capacidad de producción por proceso al mes, el desglose de la capacidad de producción en metros por hora de tela para cada una de las líneas de producto, los desperdicios estimados de tela por contracciones entre procesos por cada línea, la capacidad de almacenamiento por proceso al mes, el costo de gestión de inventarios, el nivel inicial de inventarios para el año a planificar, costos mensuales de mano de obra, eficiencia promedio en el primer mes de trabajo en empleados nuevos y los días de entrenamiento que estos requieren por proceso. Con los datos de la Tabla 3 se obtiene información de los parámetros y coeficientes necesarios para el cálculo de la capacidad de producción por proceso según la asignación de número de empleados a estos procesos.

Para ejecutar y encontrar la solución a los modelos de programación matemática de planificación agregada, existen diferentes alternativas que abarcan desde hojas de cálculo y los solvers que éstas contienen
$[1,5]$, hasta paquetes de software especializados en programación matemática tales como CPLEX [21, 22] y GAMS [14]. En el caso del modelo PLAG, se utilizó el software de optimización GAMS, el cual es un paquete informático robusto con alta capacidad de procesamiento de problemas con un número significativo de variables y restricciones. Para facilitar el ingreso de los datos de entrada del modelo en GAMS, se realizó un enlace con hojas de cálculo de MSExcel con programación en Visual Basic Applications; y de forma similar se creó una interface para facilitar la extracción, lectura y análisis de los datos de salida del modelo ejecutado. Se aclara que el modelo PLAG igualmente puede aplicarse a sectores industriales tales como el metalmecánico, maderero, confección, e inyección de plásticos, en los cuales igualmente las plantas de producción están dividas en macro procesos y se subcontratar procesos especializados de manufactura.

\section{RESULTADOS Y DISCUSIÓN}

Con base en los resultados obtenidos con el modelo PLAG se encuentra que el costo mínimo para el

Tabla 2. Demanda por línea de producto (miles de metros), horas disponibles por empleado y costos de subcontratación por mil metros de tela.

\begin{tabular}{|l|c|c|c|c|c|c|c|c|c|c|c|c|}
\hline \multirow{2}{*}{ Parámetro } & \multicolumn{10}{|c|}{ Mes $(\boldsymbol{t})$} \\
\cline { 2 - 15 } & $\mathbf{1}$ & $\mathbf{2}$ & $\mathbf{3}$ & $\mathbf{4}$ & $\mathbf{5}$ & $\mathbf{6}$ & $\mathbf{7}$ & $\mathbf{8}$ & $\mathbf{9}$ & $\mathbf{1 0}$ & $\mathbf{1 1}$ & $\mathbf{1 2}$ \\
\hline $\mathrm{D}_{l, t}$ & 72 & 84 & 84 & 102 & 96 & 72 & 84 & 108 & 132 & 96 & 90 & 84 \\
\hline $\mathrm{D}_{2, t}$ & 77 & 85 & 167 & 138 & 160 & 96 & 148 & 119 & 196 & 162 & 125 & 101 \\
\hline $\mathrm{D}_{3, t}$ & 120 & 126 & 199 & 148 & 212 & 149 & 206 & 233 & 247 & 263 & 168 & 179 \\
\hline $\mathrm{HD}_{t}$ & 206 & 174 & 200 & 206 & 200 & 200 & 216 & 200 & 208 & 208 & 192 & 190 \\
\hline $\mathrm{CSC}_{4,1, t}$ & $\$ 35,0$ & $\$ 35,3$ & $\$ 35,7$ & $\$ 36,0$ & $\$ 36,3$ & $\$ 36,7$ & $\$ 37,0$ & $\$ 37,3$ & $\$ 37,7$ & $\$ 38,0$ & $\$ 38,3$ & $\$ 38,7$ \\
\hline $\mathrm{CSC}_{4,2, t}$ & $\$ 38,7$ & $\$ 39,0$ & $\$ 39,3$ & $\$ 39,7$ & $\$ 40,0$ & $\$ 40,3$ & $\$ 40,7$ & $\$ 41,0$ & $\$ 41,3$ & $\$ 41,7$ & $\$ 42,3$ & $\$ 42,7$ \\
\hline $\mathrm{CSC}_{4,3, t}$ & $\$ 37,7$ & $\$ 38,0$ & $\$ 38,3$ & $\$ 38,7$ & $\$ 39,0$ & $\$ 39,3$ & $\$ 39,7$ & $\$ 40,0$ & $\$ 40,3$ & $\$ 41,0$ & $\$ 41,3$ & $\$ 41,7$ \\
\hline
\end{tabular}

Tabla 3. Parámetros y variables iniciales para el modelo de planificación de la producción.

\begin{tabular}{|c|c|c|c|c|r|r|r|r|r|r|r|r|r|r|r|r|}
\hline Proceso (i) & $\mathbf{P M}_{\mathbf{i}}$ & $\mathbf{M H}_{\mathbf{1}, \mathbf{i}}$ & $\mathbf{M H}_{\mathbf{2}, \mathbf{i}}$ & $\mathbf{M H}_{\mathbf{3}, \mathbf{i}}$ & $\mathbf{D T}_{\mathbf{1}, \mathbf{i}}$ & $\mathbf{D T}_{\mathbf{2}, \mathbf{i}}$ & $\mathbf{D T}_{\mathbf{3}, \mathbf{i}}$ & $\mathbf{A}_{\mathbf{i}}$ & $\mathbf{C A}_{\mathbf{i}, \mathbf{i}}$ & $\mathbf{S}_{\mathbf{1 , \mathbf { i } , \mathbf { 0 }}}$ & $\mathbf{S}_{2, \mathbf{i} \mathbf{0}}$ & $\mathbf{S}_{\mathbf{3}, \mathbf{i}, \mathbf{0}}$ & $\mathbf{C E}_{\mathbf{i}}$ & $\mathbf{X}_{\mathbf{i}, \mathbf{0}}$ & $\mathbf{E}_{\mathbf{i}}$ & $\mathbf{D E}_{\mathbf{i}}$ \\
\hline 1 & 525 & 120 & 120 & 115 & $0 \%$ & $0 \%$ & $0 \%$ & 170 & 0,011 & 20 & 10 & 15 & 607 & 13 & $72 \%$ & 5 \\
\hline 2 & 520 & 390 & 390 & 350 & $0 \%$ & $0 \%$ & $0 \%$ & 120 & 0,011 & 10 & 5 & 7 & 387 & 3 & $85 \%$ & 12 \\
\hline 3 & 490 & 219 & 219 & 240 & $3 \%$ & $3 \%$ & $2 \%$ & 50 & 0,018 & 30 & 15 & 22 & 607 & 4 & $65 \%$ & 5 \\
\hline 4 & 420 & 157 & 120 & 125 & $10 \%$ & $12 \%$ & $7 \%$ & 350 & 0,022 & 40 & 20 & 30 & 718 & 11 & $60 \%$ & 5 \\
\hline 5 & 490 & 318 & 318 & 280 & $2 \%$ & $2 \%$ & $3 \%$ & 150 & 0,022 & 20 & 10 & 15 & 387 & 3 & $75 \%$ & 12 \\
\hline $\begin{array}{l}\text { Tejeduría } \\
\text { Externa* }\end{array}$ & - & - & - & - & $12 \%$ & $15 \%$ & $9 \%$ & - & - & - & - & - & - & - & - & - \\
\hline
\end{tabular}


programa de planificación agregada para el horizonte de planificación establecido es de \$457.121 USD. Se aclara que en esta solución encontrada el $88,7 \%$ del costo total se debe al pago de mano de obra, entrenamiento de personal y costos administrativos de contratación y despido; mientras que el 5,6\% corresponde a costos de subcontratación, y el 5,7\% a costos de gestión de inventarios. Esto permite establecer que el plan de producción en el caso de estudio abordado se centra en optimizar la asignación de empleados por proceso y línea de productos por mes, así como en optimizar la contratación y despido de empleados en cada mes.

Al encontrar la solución óptima, se procedió a analizar los valores de las variables de producción por línea de producto y niveles de inventario que permiten alcanzar dicha solución al problema de planificación agregada. Para satisfacer la demanda de tela en las tres líneas de producción, el resultado del modelo de programación lineal planteado muestra estrategias diferentes para la línea de producción 1 , 2 y 3 tal y como se muestra en la Figura 1, Figura 2 y Figura 3 respectivamente.

Para la Línea 1, el resultado del modelo de programación lineal, propone una acumulación significativa de inventario en el mes 6 de 76.354 metros de tela, con los cuales se complementa la baja producción del mes 7 para satisfacer la demanda del mercado. De forma similar se comportan los meses 8 y 9 , y para los meses 10 , 11 y 12 no se acumulan inventarios, hasta el punto en que en el mes 11 y 12 se aplica una estrategia

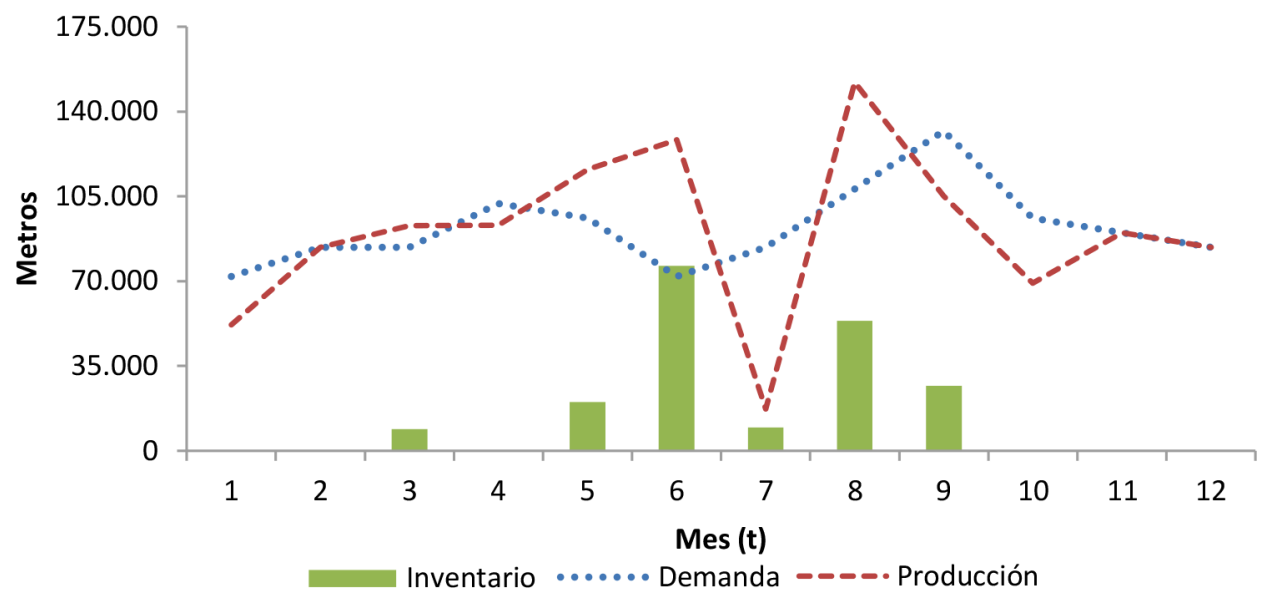

Figura 1. Demanda, producción e inventarios de tela para la Línea 1.

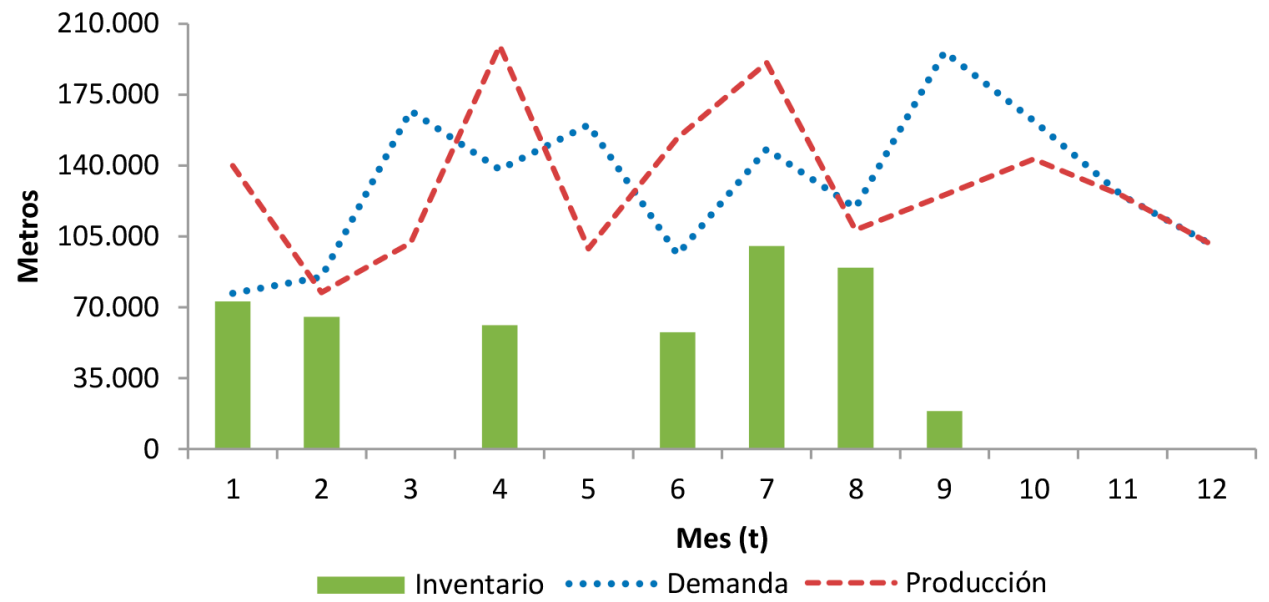

Figura 2. Demanda, producción e inventarios de tela para la Línea 2. 


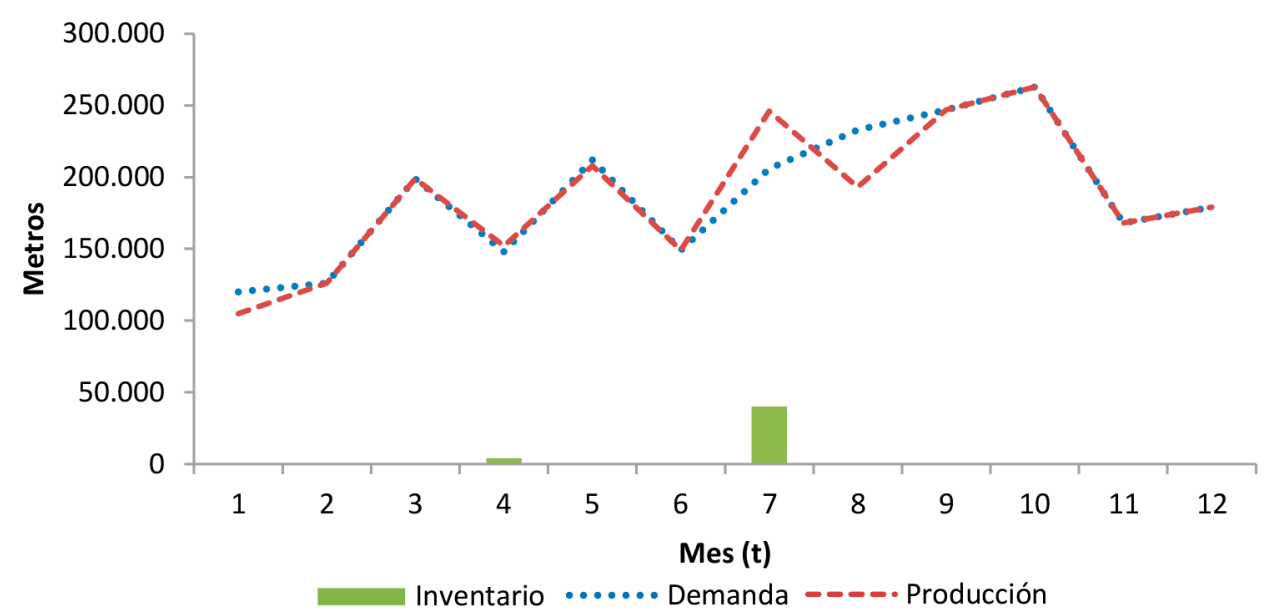

Figura 3. Demanda, producción e inventarios de tela para la Línea 3.

de seguimiento, donde la producción es igual a la demanda de mercado.

Para la Línea 2, la producción de tela es superior a la demanda de mercado para el mes 1 , por lo cual se presenta inventario de producto terminado al final del mes 1 y 2 , para luego suplir con este inventario la demanda del mes 3 , generando una reducción en la producción de tela en dicho mes. Igualmente, entre los meses 4 y 9 se presenta un comportamiento similar, en donde en ocasiones la producción supera la demanda para acumular inventarios, y luego la producción disminuye respecto a la demanda para satisfacer esta diferencia con inventarios. Al igual que sucede en la Línea 1, para la Línea 2 en el mes 11 y 12 se aplica una estrategia de seguimiento, donde la producción de tela es igual a la demanda de mercado.

Para la Línea 3, se evidencia una estrategia de seguimiento de la demanda, sin la necesidad de generar inventario de producto terminado, a excepción del mes 4 y 7 donde se genera inventario de 3.944 y 40.025 metros de tela respectivamente. Esto causa que en el mes 7 la producción sea superior a la demanda, para luego disminuir en el mes 8 la producción, y dedicar esta capacidad de producción a la Línea 1, tal y como se evidencia en la Figura 1.

Respecto a la subcontratación de producción, la Figura 4 muestra los metros de tela contratados con proveedores de servicios de manufactura para

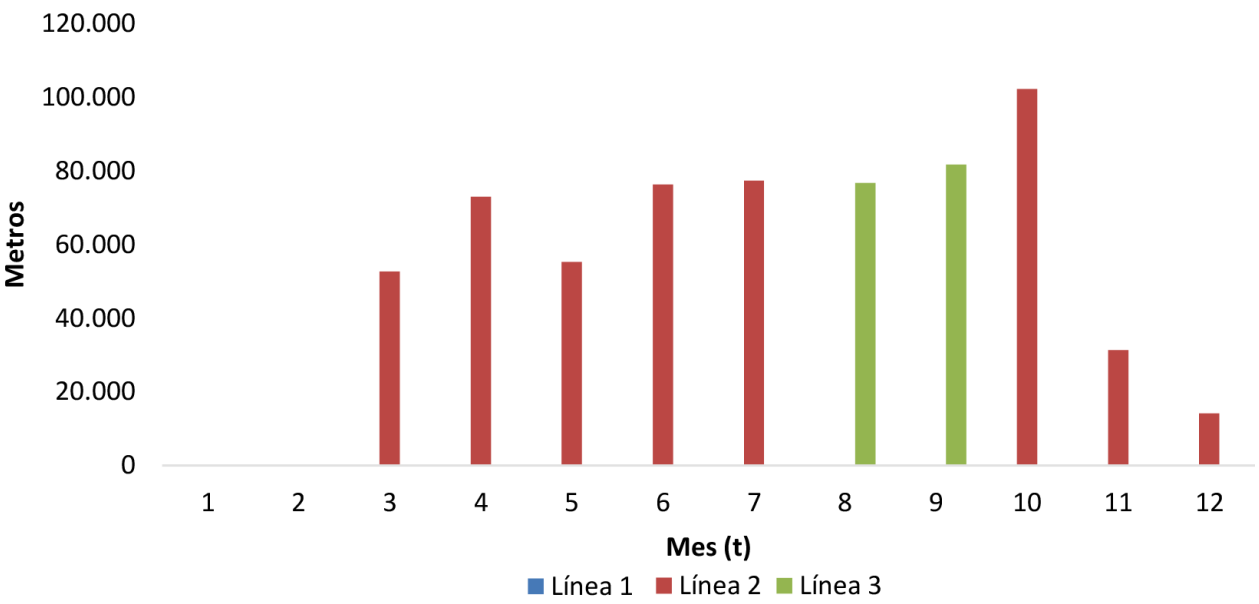

Figura 4. Producción de tela subcontratada en el proceso de tejeduría $\left(\mathrm{Y}_{i, 4, t}\right)$. 
el proceso de tejeduría para la Línea 1, Línea 2 y Línea 3. De esta forma se evidencia que para la producción de la Línea 1 no se subcontrata en ningún mes el proceso de tejeduría, mientras que en la Línea 2 es donde se subcontrata en mayor cantidad y en mayor número de meses, y en la Línea 3 solo se subcontrata producción para el mes 8 y 9. Igualmente se observa que no se presenta subcontratación para varias líneas de producto en un mismo mes, y el plan de producción sugiere un total de 641.129 metros de tela a subcontratar al año. Esta información es de gran utilidad para programar la capacidad de producción de los proveedores del proceso de tejeduría y garantizar cupos de producción entre el mes 3 y el mes 12 .

En cuanto al número de empleados por mes, contrataciones y despidos de empleados, la Figura 5 muestra los valores que permiten alcanzar la solución óptima al problema de planificación agregada. Estos resultados arrojados por el modelo PLAG sugieren en lo posible garantizar continuidad de la mano de obra en producción, mitigando así los costos de contratación y despido de la mano de obra.

De esta forma, los cambios del número de empleados se deben en mayor parte a contrataciones, para un total de 35 contrataciones en un año, teniendo mayor relevancia en el mes 3 con 23 contrataciones. Para el periodo de planificación se totalizan 14 despidos que son más representativos en el periodo 11 y 12 , con 5 y 3 despidos respectivamente debido a la disminución de las tasas de producción y generación de inventarios, que obedecen al cumplimiento de la demanda proyectada para el horizonte de planificación. Es así como, el plan de producción garantiza estabilidad en el número de empleados durante el mes 4, 5, 6, y 10, y evita despidos de empleados durante 6 meses del año.

Se aclara también de la Figura 5 que para el mes 1 se presentan tanto contrataciones como despidos debido a que se contrata 1 persona para el proceso de engomado y 3 personas para el proceso de revisión de tejido, mientras se despiden 3 personas del proceso de urdido. De forma similar, en el mes 3 se presentan contrataciones y despidos de empleados debido a que se contratan 11 personas para el proceso urdido, 3 personas para el proceso de revisión de urdido, 3 personas para el proceso de engomado, 3 personas para el proceso de tejido y 2 personas para el proceso de revisión de tejido, mientras se despide 1 persona del proceso de urdido y 1 persona del proceso de engomado. Para el caso del mes 3 , se explica que los empleados que se despidieron del proceso de urdido y engomado solo trabajaron durante dicho mes.

En general, el plan de producción agregada creado por el modelo PLAG propone el uso de inventarios de producto en proceso y producto terminado, subcontratar el proceso de tejeduría para la Línea 2 y Línea 3, además de realizar las mínimas contrataciones y despidos posibles debido

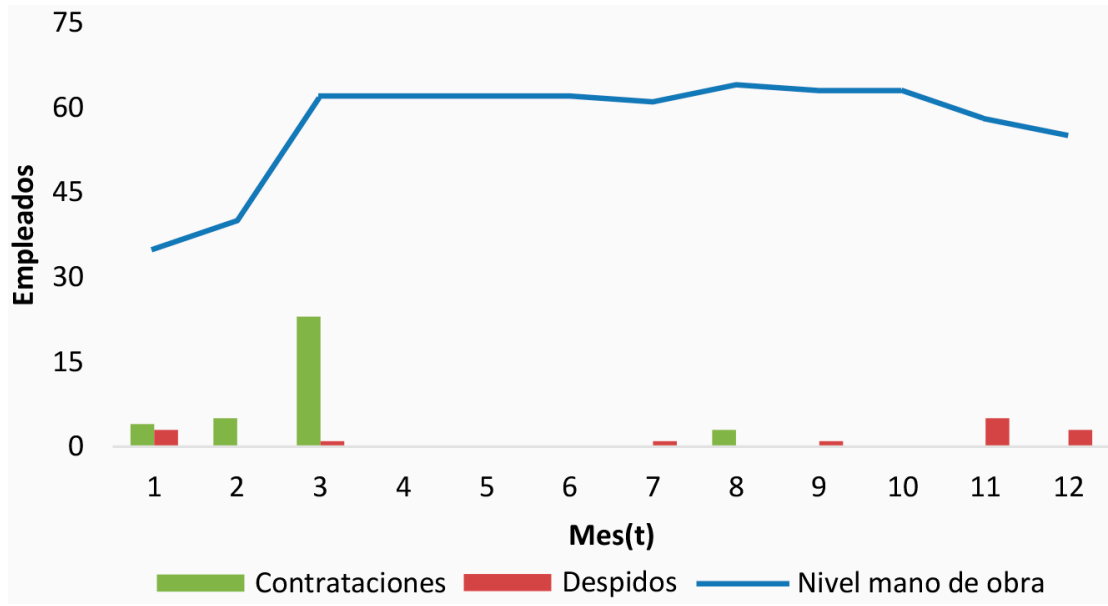

Figura 5. Nivel de mano de obra $\left(\mathrm{X}_{i, t}\right)$, contrataciones $\left(\mathrm{XN}_{i, t}\right)$ y despidos $\left(\mathrm{XD}_{i, t}\right)$ por línea y mes. 
a los altos costos que estas generan. Por lo tanto, tal y como se evidencia en la Figura 6, no existen grandes diferencias entre la demanda acumulada y la producción acumulada, sugiriendo la acumulación de inventario principalmente para los meses 6 , 7, 8 y 9 a través del incremento de producción respecto a la demanda, para luego disminuir la tasa de producción respecto a la demanda en el último trimestre del periodo de planificación, con el fin de reducir los altos costos de subcontratación de producción, contratación, entrenamiento y despido que implicaría tener niveles de producción variables.

Estas decisiones se refuerzan con el análisis de los costos reducidos de las variables no básicas de inventario, los cuales presentan valores elevados, que implican que al almacenar un metro de tela por un mes aumenta el costo total por metro hasta en $\$ 0,19$ USD. De forma similar, el costo de contratar a un empleado diferente a lo que sugiere la solución óptima, puede aumentar el costo total del plan de producción hasta en \$544 USD. Es entonces, así como en los últimos meses se eliminan los inventarios finales, no se realizan contrataciones, y se generan despidos, obteniendo el menor costo posible, y satisfaciendo las condiciones y restricciones planteadas en el modelo.

Al comparar los resultados del modelo PLAG con los resultados obtenidos con el modelo propuesto por Campo et al. [19], se observa que este último modelo genera una proporción de costos de mano de obra sobre el costo total del $95 \%$, mientras que en el caso del modelo PLAG esta proporción es del $88,7 \%$ debido a la consideración de subcontratación del proceso de tejeduría, que alcanza a representar un $5,6 \%$ del costo total. Respecto a los costos de gestión de inventarios, se identifica que ambos modelos presentan proporciones cercanas al 5\% sobre el costo total, confirmando así que la flexibilidad que brinda la subcontratación de procesos de manufactura permite generar ahorros en mano de obra y en el costo total.

\section{Análisis de sensibilidad}

Para complementar el análisis de la solución óptima encontrada con el modelo PLAG, se analizan las restricciones de almacenamiento y de capacidad de producción por proceso (ya que son las restricciones que realmente cambian la solución obtenida), identificando los precios sombra diferentes a cero y los intervalos en los cuales se pueden modificar estos parámetros, de tal forma que la solución encontrada siga siendo óptima. Este análisis se realiza a partir de los datos arrojados por el software GAMS donde se ejecutó el modelo propuesto, obteniendo en la Tabla 4 los precios sombra diferentes de cero para las restricciones de capacidad de almacenamiento.

De la Tabla 4 se deduce que al aumentar la capacidad de almacenamiento del proceso 3 en el mes 5 en

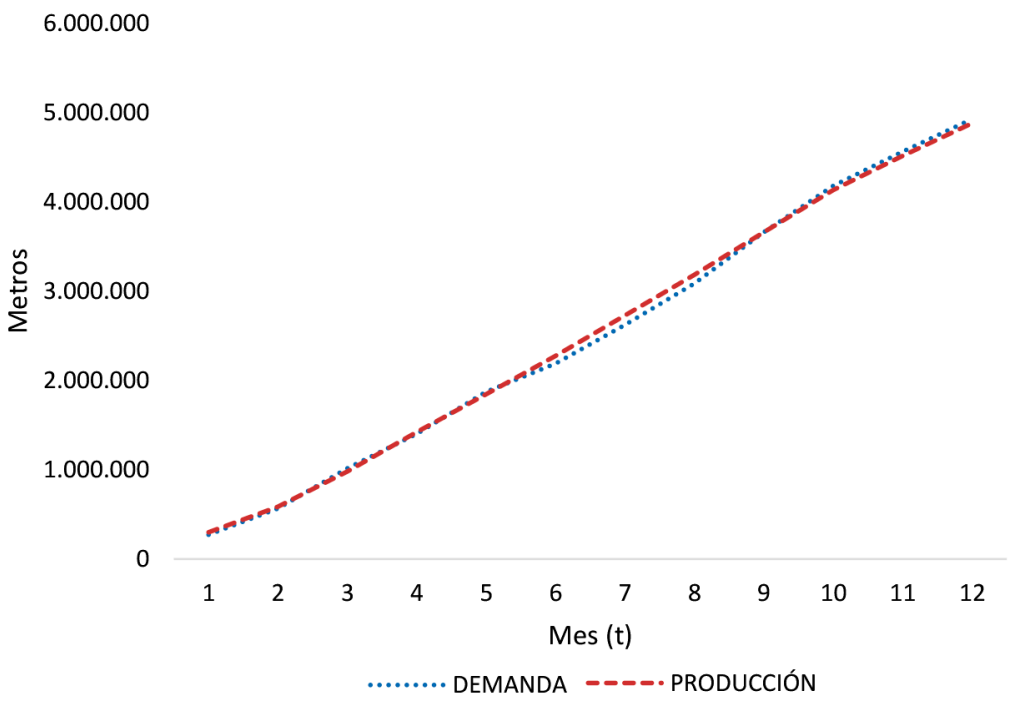

Figura 6. Demanda y producción acumulada para el plan agregado de producción. 
Tabla 4. Análisis de sensibilidad para la capacidad de almacenamiento.

\begin{tabular}{|c|c|c|c|c|c|c|}
\hline Proceso (i) & Mes (t) & $\begin{array}{c}\text { Capacidad } \\
\text { disponible } \\
\text { (Lado derecho) }\end{array}$ & $\begin{array}{c}\text { Capacidad de } \\
\text { almacenamiento } \\
\text { utilizada }\end{array}$ & $\begin{array}{c}\text { Intervalo } \\
\text { inferior }\end{array}$ & $\begin{array}{c}\text { Intervalo } \\
\text { superior }\end{array}$ & $\begin{array}{c}\text { Precio sombra por } \\
\text { cada aumento en } \\
\mathbf{1 . 0 0 0} \text { metros }\end{array}$ \\
\hline \multirow{3}{*}{3} & 5 & 30.000 & 30.000 & 27.370 & 39.960 & $-\$ 0,15$ \\
\cline { 2 - 7 } & 6 & 30.000 & 30.000 & 8.377 & 48.670 & $-\$ 4,76$ \\
\cline { 2 - 7 } & 7 & 30.000 & 30.000 & 20.390 & 55.360 & $-\$ 5,53$ \\
\hline 5 & 8 & 30.000 & 30.000 & 0 & 54.300 & $-\$ 4,74$ \\
\hline
\end{tabular}

1.000 metros, los costos totales (función objetivo) disminuirán en \$0,15 USD, y esto será proporcional por cada 1.000 metros de aumento en capacidad de almacenamiento, siempre y cuando dicha capacidad no sobrepase 39.600 metros. Por lo tanto, por cada aumento en 1.000 metros de la capacidad de almacenamiento desde el mes 5 hasta el mes 8 en el proceso 3 , los costos totales disminuirán aproximadamente $\$ 15,17$ USD, siempre y cuando la capacidad de almacenamiento 3 no sobrepase los 39.600 metros, ya que al superar esta capacidad de almacenamiento se disminuirán los costos totales en $\$ 15,02$ USD por cada 1.000 metros de aumento de capacidad. Del mismo modo, si la capacidad de almacenamiento sobrepasa los 48.670 metros, los costos totales disminuirán en \$10,26 USD, y así sucesivamente. Otro aumento de capacidad de almacenamiento que generaría disminución en los costos totales, sería en el mes 8 para el proceso 5 , ya que por cada 1.000 metros que se aumente dicha capacidad se disminuirán los costos en aproximadamente $\$ 19,93$ USD.

En la Tabla 5 se presentan los costos que se pueden reducir al realizar diferentes aumentos de capacidad de almacenamiento en los procesos 3 y 5 . De esta forma, si se aumenta la capacidad de almacenamiento del proceso 3 desde el mes 5 en 9.960 metros de tela, es decir, pasar de 30.000 a 39.960 metros, la reducción en costo total será de $\$ 151,08$ USD, pero si el aumento es de 18.670 metros, la reducción en costo será de \$281,94 USD. Igualmente, se observa que al aumentar la capacidad de almacenamiento del proceso 3 desde el mes 5 en 25.360 metros, se generaría una reducción del costo total de $\$ 344,74$ USD, y que a partir de ese punto no es necesario realizar aumentos en la capacidad de almacenamiento del proceso 3, ya que no generaría reducción del costo total. De forma similar, un aumento de hasta 8.600 metros en el proceso 5 en el mes 8 aportaría a una reducción de \$171,39 USD en el costo total.

Del mismo modo, con los datos arrojados por el software GAMS se realiza un análisis de sensibilidad para las restricciones de capacidad de producción en los procesos de manufactura, presentando en la Tabla 6 los precios sombra diferentes de cero para las restricciones de capacidad de producción. De allí se destaca que los procesos 1, 2, 3 y 4 son sensibles a mejorar el costo total, pero de estos procesos el

Tabla 5. Cálculo de reducción de costo total según aumento de capacidad de almacenamiento.

\begin{tabular}{|c|c|c|c|c|c|}
\hline \multirow{2}{*}{$\begin{array}{c}\text { Proceso } \\
\text { (i) }\end{array}$} & $\begin{array}{c}\text { Capacidad } \\
\text { actual de } \\
\text { almacenamiento }\end{array}$ & \multicolumn{2}{|c|}{$\begin{array}{c}\text { Intervalo para el } \\
\text { aumento de capacidad } \\
\text { de almacenamiento }\end{array}$} & $\begin{array}{c}\text { Reducción de costo total } \\
\text { por cada aumento de } \\
\mathbf{1 . 0 0 0} \text { metros }\end{array}$ & $\begin{array}{c}\text { Reducción } \\
\text { máxima de costo } \\
\text { total }\end{array}$ \\
\cline { 3 - 4 } & & Lím. Inf. & Lím. Sup. & & \\
\hline \multirow{3}{*}{3} & \multirow{3}{*}{30.000} & 0 & 9.960 & $15,17 \mathrm{X}$ & $\$ 151,08$ \\
\cline { 3 - 6 } & & 9.960 & 18.670 & $151,08+15,02(\mathrm{X}-9,96)$ & $\$ 281,94$ \\
\cline { 3 - 6 } & & 18.670 & 24.300 & $281,94+10,26(\mathrm{X}-18,67)$ & $\$ 339,72$ \\
\cline { 3 - 6 } & 150.000 & 24.300 & 25.360 & $339,72+4,74(\mathrm{X}-24,3)$ & $\$ 344,74$ \\
\hline 5 & 0 & 8.600 & $19,92 \mathrm{X}$ & $\$ 171,39$ \\
\hline
\end{tabular}

$\mathrm{X}$ : Aumento en miles de metros en la capacidad de almacenamiento. 
Tabla 6. Análisis de sensibilidad para la capacidad de producción.

\begin{tabular}{|c|c|c|c|c|c|c|}
\hline Proceso (i) & Mes (t) & $\begin{array}{c}\text { Capacidad } \\
\text { disponible } \\
\text { (Lado derecho) }\end{array}$ & $\begin{array}{c}\text { Capacidad de } \\
\text { producción } \\
\text { utilizada }\end{array}$ & $\begin{array}{c}\text { Intervalo } \\
\text { inferior }\end{array}$ & $\begin{array}{c}\text { Intervalo } \\
\text { superior }\end{array}$ & $\begin{array}{c}\text { Precio sombra } \\
\text { por cada aumento } \\
\text { en 1.000 metros }\end{array}$ \\
\hline 1 & 7 & 525.000 & 525.000 & 525.000 & 526.100 & $\$ 62,01$ \\
\hline \multirow{3}{*}{2} & 4 & 520.000 & 520.000 & 518.200 & 520.600 & $\$ 1,49$ \\
\cline { 2 - 7 } & 7 & 520.000 & 520.000 & 517.300 & 524.500 & $\$ 17,42$ \\
\hline \multirow{4}{*}{3} & 4 & 510.000 & 510.000 & 508.300 & 518.000 & $\$ 52,48$ \\
\cline { 2 - 7 } & 5 & 510.000 & 510.000 & 507.900 & 511.500 & $\$ 78,94$ \\
\cline { 2 - 7 } & 6 & 510.000 & 510.000 & 509.100 & 516.600 & $\$ 58,41$ \\
\cline { 2 - 7 } & 7 & 510.000 & 510.000 & 508.400 & 514.200 & $\$ 147,21$ \\
\cline { 2 - 7 } & 8 & 510.000 & 510.000 & 508.400 & 517.400 & $\$ 108,75$ \\
\hline \multirow{4}{*}{4} & 10 & 510.000 & 510.000 & 510.000 & 512.000 & $\$ 241,32$ \\
\hline & 4 & 420.000 & 420.000 & 417.700 & 421.600 & $\$ 277,35$ \\
\hline & 6 & 420.000 & 420.000 & 415.700 & 431.500 & $\$ 23,12$ \\
\hline & 7 & 420.000 & 420.000 & 415.700 & 431.500 & $\$ 27,85$ \\
\hline & 8 & 420.000 & 420.000 & 383.700 & 426.700 & $\$ 32,54$ \\
\hline & 9 & 420.000 & 420.000 & 391.200 & 426.700 & $\$ 37,11$ \\
\hline & 10 & 420.000 & 420.000 & 413.800 & 426.400 & $\$ 41,64$ \\
\hline
\end{tabular}

proceso 3 puede generar mayor reducción del costo total al aumentar su capacidad de producción en el mes 10 , ya que por cada 1.000 metros que se aumente la capacidad de dicho proceso, el costo total se reducirá en $\$ 277,35$ USD, e incluso si la capacidad de producción del proceso 3 en el mes 10 aumenta 5.100 metros (pasar de 510.000 a 515.100 metros), la reducción del costo total será de $\$ 1.414,51$ USD.

En este sentido, en la Tabla 7 se muestran los costos que pueden reducirse al aumentar la capacidad de producción en los procesos que presentan precios sombras diferentes a cero, especialmente para el proceso 3 y 4 que son los más sensibles a cambios. Para la restricción de capacidad de producción se observa que el proceso que generaría mayor reducción de costos al aumentarse su capacidad de producción es el proceso 3, alcanzando un ahorro hasta de $\$ 3.648,38$ USD si su capacidad de producción se aumenta en 8.000 metros a partir del mes 4. Igualmente, se puede reducir el costo total en \$1.196,33 USD si se aumenta en 11.500 metros la capacidad de producción del proceso 4 a partir del mes 4 .
De esta forma, el análisis de sensibilidad permite establecer estrategias de producción que priorizan el aumento de la capacidad de producción sobre el aumento de capacidad de almacenamiento por proceso. Específicamente, el aumento de capacidad de producción en un solo proceso puede generar reducción de costos de hasta $\$ 3.648,38 \mathrm{USD}$, mientras que el aumento de capacidad de almacenamiento de un solo proceso generaría una reducción máxima de $\$ 344,74$ USD en el costo total.

Finalmente, al aplicar las mejoras pertinentes al plan agregado de producción y autorizar su ejecución, se debe proceder con la integración de la programación de mediano y corto plazo, a través de sistemas MRP [23] y sistemas de programación de la producción [24] que permiten el despliegue de estrategias de producción en decisiones tácticas y operativas en ambientes de manufactura.

\section{CONCLUSIONES}

El desarrollo de modelos de programación matemática para la planificación de la producción agregada, 
Tabla 7. Cálculo de reducción de costo total según aumento de capacidad de producción.

\begin{tabular}{|c|c|c|c|c|c|}
\hline \multirow{2}{*}{ Proceso (i) } & \multirow{2}{*}{$\begin{array}{l}\text { Capacidad } \\
\text { actual de } \\
\text { producción }\end{array}$} & \multicolumn{2}{|c|}{$\begin{array}{l}\text { Intervalo para el aumento } \\
\text { de capacidad de producción }\end{array}$} & \multirow{2}{*}{$\begin{array}{c}\text { Reducción de costo total } \\
\text { por cada aumento de } \\
1.000 \text { metros }\end{array}$} & \multirow{2}{*}{$\begin{array}{l}\text { Reducción } \\
\text { máxima de } \\
\text { costo total }\end{array}$} \\
\hline & & $\begin{array}{l}\text { Límite } \\
\text { inferior }\end{array}$ & $\begin{array}{l}\text { Límite } \\
\text { superior }\end{array}$ & & \\
\hline \multirow{7}{*}{3} & \multirow{7}{*}{510.000} & 0 & 1.500 & $964,46 \mathrm{X}$ & $\$ 1.446,69$ \\
\hline & & 1.500 & 2.000 & $1446,69+885,52(X-1,5)$ & $\$ 1.889,45$ \\
\hline & & 2.000 & 4.200 & $1889,45+644,2(X-2)$ & $\$ 3.306,70$ \\
\hline & & 4.200 & 5.100 & $3306,7+497(X-4,2)$ & $\$ 3.377,74$ \\
\hline & & 5.100 & 6.600 & $3377,74+219,64(X-5,1)$ & $\$ 3.465,36$ \\
\hline & & 6.600 & 7400 & $3465,36+161,23(X-6,6)$ & $\$ 3.583,13$ \\
\hline & & 7.400 & 8000 & $3583,13+52,48(X-7,4)$ & $\$ 3.648,38$ \\
\hline \multirow{4}{*}{4} & \multirow{4}{*}{420.000} & 0 & 1.600 & $168,91 \mathrm{X}$ & $\$ 270,25$ \\
\hline & & 1.600 & 6.400 & $270,25+162,27(X-1,6)$ & $\$ 1.049,15$ \\
\hline & & 6.400 & 6.700 & $1049,15+120,63(X-6,4)$ & $\$ 1.085,34$ \\
\hline & & 6.700 & 11.500 & $1085,34+50,98(X-6,7)$ & $\$ 1.196,33$ \\
\hline
\end{tabular}

$\mathrm{X}$ : Aumento en miles de metros en la capacidad de almacenamiento.

no solo se limita a generar una solución óptima para ejecutar un plan de producción con base en las condiciones actuales de un proceso productivo, sino que también permite identificar estrategias de mejoramiento para la disminución de costos totales tales como aumento de capacidad de producción, aumento de almacenamiento por proceso, negociación con proveedores para la subcontratación de producción, y variación de la mano de obra en cada periodo de planificación.

A través de modelos de programación lineal como el PLAG, se logra representar las condiciones reales y particulares de un proceso productivo, modificando fácilmente los parámetros por parte de quienes administran la producción y toman decisiones estratégicas en la gestión de operaciones. Del mismo modo, se logra respaldar el proceso de toma de decisiones a través de resultados cuantitativos para satisfacer objetivos empresariales y cumplir con restricciones intrínsecas de procesos y sectores empresariales tales como la industria textil. De igual forma, el modelo PLAG desarrollado contribuye a la creación de modelos que se ajustan más a la realidad del negocio, debido a que tiene en cuenta variables que afectan de forma significativa el desempeño de un plan de producción del sector textil, tales como contracciones de tela, pérdidas por manipulación del producto en el proceso, eficiencia de empleados nuevos, tiempos de entrenamiento y capacitación, y subcontratación de procesos de manufactura.
Para el caso de estudio tratado en este artículo, la estrategia de producción óptima arrojada por el modelo PLAG es una estrategia mixta, que en algunas líneas de producción para determinados periodos de tiempo sugiere realizar estrategias de nivel o equilibrio y estrategias de alcance con base en inventario, subcontratación de producción, contrataciones y despidos de mano de obra. De forma similar, para disminuir los costos y alcanzar las metas de producción, el modelo sugiere como principal estrategia aumentar la capacidad de producción del proceso 3 (Engomado) en 8.000 metros de tela, a partir del mes 4, para aumentar así la capacidad total del proceso productivo y reducir los costos totales del plan de producción en \$3.648,38 USD. Del mismo modo, los resultados del modelo PLAG plantean que en caso de aumentarse la demanda de mercado es necesario aumentar la capacidad de producción y/o la capacidad de almacenamiento del proceso 3 (Engomado), ya que este se identificó como el proceso restrictivo o cuello de botella.

Con base en los resultados, se recomienda subcontratar el proceso de tejeduría únicamente para la Línea 2 y Línea 3, lo cual permite minimizar costos, realizar una negociación constante con los proveedores de procesos de manufactura y disminuir la variabilidad en la contratación y despido de mano de obra. Finalmente, como líneas futuras de investigación se sugiere el modelamiento de algunos parámetros con lógica difusa $[23,25]$ para considerar incertidumbre 
en el modelo PLAG y representar de mejor forma condiciones reales de ambientes de fabricación y manufactura.

\section{REFERENCIAS}

[1] B.H. Simamora and D. Natalia. "Aggregate Planning for Minimizing Costs: A Case Study of PT XYZ in Indonesia". International Business Management. Vol. 8, Issue 6, pp. 353-356. 2014. DOI: 10.3923/ ibm.2014.353.356.

[2] R. Zhang, P.-C. Chang, S. Song and C. Wu. "Local search enhanced multi-objective PSO algorithm for scheduling textile production processes with environmental considerations". Applied Soft Computing Journal. Vol. 61, Issue 1, pp. 447-467. 2017. DOI: 10.1016/j. asoc.2017.08.013.

[3] M. Sadeghi, S.H. Razavi Hajiagha and S.S. Hashemi. "A fuzzy grey goal programming approach for aggregate production planning". The International Journal of Advanced Manufacturing Technology. Vol. 64, Issue 9-12, pp. 1715-1727. 2013. DOI: 10.1007/s00170-012-4135-y.

[4] A. Alfieri, T. Tolio and M. Urgo. "A project scheduling approach to production and material requirement planning in Manufacturing-to-Order environments". Journal of Intelligent Manufacturing. Vol. 23 $\mathrm{N}^{\circ}$ 3, pp. 575-585. 2012. DOI: 10.1007/ s10845-010-0396-1.

[5] R.L. Espey and J. Balakrishnan. "A spreadsheet decision support optimization model for railcar storage at Canadian Pacific Railway". Journal of the Operational Research Society. Vol. 63, Issue 2, pp. 139-150. 2012. DOI: 10.1057/jors.2010.178.

[6] M. Mezghani and T. Loukil. "Remanufacturing planning with imprecise quality inputs through the Goal Programming and the satisfaction functions". International Journal of Multicriteria Decision Making. Vol. 2, Issue 4, pp. 379-390. 2012. DOI: 10.1504/ IJMCDM.2012.050680.

[7] A. Anand, C. Krishnaraj and S.R. Kasthuri. "LINGO based revenue maximization using aggregate planning." ARPN Journal of Engineering and Applied Sciences. Vol. 11, Issue 9, pp. 6075-6081. 2016. ISSN: 1819-6608.
[8] G. Buxey. "Aggregate planning for seasonal demand: reconciling theory with practice". International Journal of Operations \& Production Management. Vol. 25, Issue 11, pp. 1083-1100. 2005. DOI: 10.1108/01443570510626907.

[9] P. Ji and K. Chu. "A dual-matrix approach to the transportation problem". Asia-Pacific Journal of Operational Research. Vol. 19, Issue 1, pp. 35-45. 2002. ISSN: 0217-5959.

[10] A. Lusa, C. Martínez-Costa and M. MasMachuca. "An integral planning model that includes production, selling price, cash flow management and flexible capacity". International Journal of Production Research. Vol. 50, Issue 6, pp. 1568-1581. 2012. DOI: 10.1080/00207543.2011.558128.

[11] J. Arts and S.D. Flapper. "Aggregate overhaul and supply chain planning for rotables". Annals of Operations Research. Vol. 224, Issue 1, pp. 77-100. 2015. DOI: 10.1007/ s10479-013-1426-0.

[12] C. Gomes da Silva, J. Figueira, J. Lisboa and S. Barman. "An interactive decision support system for an aggregate production planning model based on multiple criteria mixed integer linear programming". Omega. Vol. 34, Issue 2, pp. 167-177. 2006. DOI: 10.1016/J.OMEGA.2004.08.007

[13] A. Corominas, A. Lusa and J. Olivella. "A manufacturing and remanufacturing aggregate planning model considering a non-linear supply function of recovered products". Production Planning \& Control. Vol. 23, Issue 2-3, pp. 194-204. 2012. DOI: 10.1080/09537287.2011.591651.

[14] R. de Á.R. Junqueira and R. Morabito. "Um modelo de otimização linear para o planejamento agregado da produção e logística de sementes de milho". Production. Vol. 16, Issue 3, pp. 510-525. 2006. DOI: 10.1590/S0103-65132006000300012.

[15] M.G. Jennings and N. Shah. "Workforce planning and technology installation optimisation for utilities." Computers \& Industrial Engineering. Vol. 67, Issue 1, pp. 72-81. 2014. DOI: 10.1016/J. CIE.2013.10.008.

[16] S. Plonka and L. Oginski. "Multicriterial Optimisation of the Manufacturing Process of a Spindle Working in a Ring Spinning 
Frame". Fibres \& Textiles in Eastern Europe. Vol. 22, Issue 6, pp. 51-58. 2014. ISSN: 1230-3666.

[17] H.J. Mercado, T.J. Fontalvo and E.H. Granadillo. "Análisis comparativo entre las cadenas productivas del sector textilconfecciones de la provincia de Jiangsu-China y el departamento del Atlántico-Colombia". Ingeniare. Revista Chilena de Ingeniería. Vol. $19 \mathrm{~N}^{\circ} 3$, pp. 429-441. 2011. DOI: 10.4067/S0718-33052011000300012.

[18] J.J. Lopes, M.L.R. Varela, J. Trojanowska and J. Machado. "Production flow improvement in a textile industry". Advances in Intelligent Systems and Computing. Vol. 721, Issue 1, pp. 224-233. 2018. DOI: 10.1007/978-3-319-73450-7_22.

[19] E.A. Campo, J.A. Cano and R.A. GómezMontoya. "Linear Programming for Aggregate Production Planning in a Textile Company," Fibres \& Textiles in Eastern Europe. Vol. 5, Issue 131, pp. 13-20. 2018. DOI: 10.5604/01.3001.0012.13XX.

[20] A. Zuluaga, J.A. Cano and M. Montoya. "Gestión logística en el sector textilconfección en Colombia: retos y oportunidades de mejora para la competitividad". Revista Clío América. Vol. 12, Issue 23, pp. 1-10. 2018. ISSN: ISSN 2389-7848.
[21] A. Jain and U.S. Palekar. "Aggregate production planning for a continuous reconfigurable manufacturing process". Computers \& Operations Research. Vol. 32, Issue 5, pp. 1213-1236. 2005. DOI: 10.1016/J. COR.2003.11.001.

[22] R. Linfati-Medina, L. Pradenas-Rojas and J. Ferland. "Planificación agregada en la cosecha forestal: Un modelo de programación matemática y solución". Maderas: Ciencia y Tecnología. Vol. $18 \mathrm{~N}^{\circ}$ 4, pp. 555-566. 2016. DOI: $10.4067 / \mathrm{S} 0718-221 X 2016005000048$.

[23] M. Arango, J.A. Cano and K. Alvarez. "Modelos de sistemas MRP cerrados integrando incertidumbre." Revista EIA. Vol. $18 \mathrm{~N}^{\circ}$ 1, pp. 61-76. 2012. ISSN 1794-1237.

[24] J.A. Cano, E.A. Campo and R. Gómez. "Simulación de eventos discretos en la planificación de producción para sistemas de confección modular". Revista Técnica de la Facultad de Ingeniería Universidad del Zulia. Vol. $41 \mathrm{~N}^{\circ}$ 1, pp. 50-58. 2018. ISSN 0254-0770.

[25] R. Gómez, J.A. Cano and E.A. Campo. "Selección de proveedores en la minería de oro con lógica difusa". Revista Venezolana de Gerencia (RVG). Vol. 21 N $^{\circ} 75$, pp. 530548. 2018. ISSN 1315-9984. 